\title{
Characteristics Analysis of Chemical Concentration Sensor Based on Three-layer FBG
}

\author{
Zhaoxia Wu, Xinyan Yu, Erdan Gu, Zhi Kong, Wenchao Li \\ College of Control Engineering, Northeastern University at Qinhuangdao, Qinhuangdao Hebei 066004, China \\ Email: ysuwzx@126.com
}

Received 2013

\begin{abstract}
The three-layer structure model of the fiber Bragg grating is proposed in this paper. And through experimental study and reasoning calculation to verify the three layer structure of the optical waveguide model, so the sensitivity characteristic of the Bragg wavelength to the refractive index of chemical solution is obtained. And the relationships between the concentration and the shift of Bragg wavelength of sucrose, ethanol, and Nacl solution are achieved. Finally the shifts of the Bragg wavelength with the external concentration are obtained by experiments. A kind of simple structure, small size, good stability, high sensitivity chemical concentration sensor is obtained.
\end{abstract}

Keywords: Fiber Bragg grating(FBG); Three-layer Structure; Refractive Index; Concentration of Solution; Chemical Sensor

\section{Introduction}

The concentration of solution is considered as one of the integrated indicators of medium solution. The concentration measurement and control of solution is widely used on papermaking, chemical, sugar production, food industry, environmental monitoring, etc. It is the important technical means guarantee product quality and improve the quality of products. Fiber Bragg grating (FBG) has many virtues such as resistant to electromagnetic interference, high insulating, corrosion resistance small bulk, low weight, high sensitivity, absolute code of wavelength, compare with fiber, easy to reuse into network, etc. The liquid concentration sensor takes FBG as its sensitive element has positive research significance and potential application prospect $[1,2]$. The fiber Bragg grating sensor plays an important role in the measurement of liquid substance. It can distinguish the content of measured substance by measuring wavelength change of reflection center [3]. Therefore, improve the sensitivity of the fiber Bragg grating on the refractive index of the surrounding medium in the existing technical is the key [4]. At present there are mainly two kinds of fiber grating sensors used for measuring the refractive index change, fiber Bragg grating (FBG) and long period fiber grating (LPFG) [5]. As a result of the coupling based on the core mode and cladding mode, the external refractive index change will have a greater impact on fiber cladding mode transmission properties; therefore the long period fiber grating has a larger sensitivity. But the long period fiber grating being very sensitive to optical fiber bending and laying state, the high order cladding mode transmission properties being unstable, and its multi-Peak Value, large bandwidth and the working mode of using the transmission spectrum only to detect limit its measuring precision and multiplexing capability[6]. Currently, the refractive index sensor based on fiber Bragg grating has been reported [7, 8], but there are no in-depth theoretical analysis, numerical calculations and experiment contrast results. This paper focuses on the FBG chemical sensor's theoretical analysis and establishes the three-layer structure model, while the three solutions of different concentrations were experimentally, and realize the high sensitivity measurement of chemical solution concentration. The sensor doesn't need coating intermediate sensitive material, can measure various chemical solution concentrations. The sensor has the advantages of simple structure, high precision, low cost, eases of reuse, can realize on-line measurement and so on. Also it has broad application prospects.

\section{Theoretical Mode}

Based on the fiber grating coupled mode theory and the phase matching condition, the wavelength $\lambda_{B}$ of FBG is:

$$
\lambda_{\mathrm{B}}=2 \Lambda n_{\text {eff }}
$$

where, $n_{\text {eff }}$ is the effective refractive index of core region of the fiber, $\Lambda$ is the grating period. 
As the cladding thickness decreases (usually a few microns), evanescent waves project through the cladding layer. Under the effect of the evanescent wave, the effective refractive index of fibers and solution index have a dependency relation [9]. The refractive index of solution changes with the change of the concentration of solution. Bragg wavelength changes with the change of the effective refractive index. So the response to the concentration of chemical solution can realize by measuring the Bragg wavelength to reflect the change of the concentration of chemical solution.

First, discussed the changes of the external refractive index SRI ranged from 1.32 riu to 1.46 riu and its effect on FBG effective refractive index: suppose, $\lambda$ is the operating wavelength of $\mathrm{LP}_{01}, \mathrm{n}_{\text {eff }}$ is the refractive index of $\mathrm{LP}_{01}$. And it is shown in Figure 1. The $\mathrm{LP}_{01}$ model's characteristic equation can be expressed as:

$$
\begin{gathered}
{\left[J_{0}(u)-K_{0}\left(v^{\prime} c\right)\right] \times\left[K_{0}(v)+\hat{I}_{0}\left(v^{\prime}\right)\right] /} \\
{\left[K_{0}(v)-K_{0}\left(v^{\prime}\right)\right] /\left[J_{0}(u)+\hat{I}_{0}\left(v^{\prime} c\right)\right]=} \\
I_{1}\left(v^{\prime} c\right) K_{1}\left(v^{\prime}\right) /\left[I_{1}\left(v^{\prime}\right) K_{1}\left(v^{\prime} c\right)\right] \\
u=(2 \pi / \lambda) a\left(n_{1}^{2}-n_{\text {eff }}^{2}\right)^{1 / 2} \\
v=(2 \pi / \lambda) b\left(n_{\text {eff }}^{2}-S_{R I}^{2}\right)^{1 / 2} \\
v^{\prime}=(2 \pi / \lambda) b\left(n_{\text {eff }}^{2}-n_{2}^{2}\right)^{1 / 2} \\
c=a / b
\end{gathered}
$$

where, $a$ is the fiber radius, $b$ is the cladding radius after etching, $n_{1}$ and $n_{2}$ is respectively the core and the cladding refractive index, $J$ is the Bessel function of the first kind, $I$ is the variant Bessel function of the first kind, $K$ is the variant Bessel function of the second kind.

The reflectivity and transmissivity expression of the Fiber Bragg grating are deduced through coupled mode equation and boundary condition of Fibber Bragg grating, in the phase matching condition, corresponding to the maximum reflectivity and transmissivity:

$$
R_{\max }=\tanh ^{2}(S L)=\tanh ^{2}\left(\frac{\pi \Delta n}{\lambda_{B}} L\right)
$$

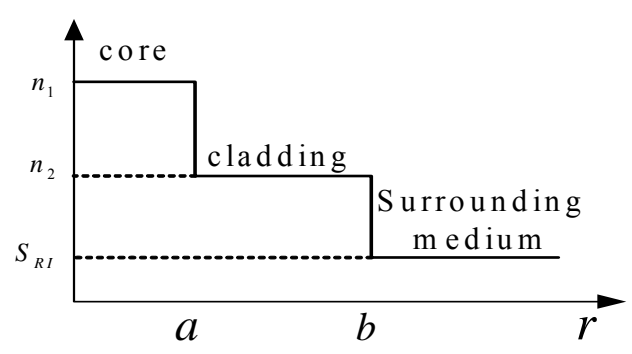

Figure1. Optical waveguide model of three-layer structure.

$$
T_{\text {max }}=\operatorname{arcosh}(S L)
$$

Where $S=\sqrt{k^{2}-(\Delta \beta)^{2}}, k$ is the coupling coefficient, $\Delta \beta$ is phase-mismatching condition.

So, the reflectance spectrum between Bragg reflection wavelength and change of SRI offset size is obtained through the reflective index change of solution. It can be more clearly viewed how the external solutions influence the Fiber Bragg grating center wavelength.

\section{The Simulation Results}

For the specific fiber grating, solutions meeting equation (1) - (6) can be found if given the SRI range. And the solutions are just the FBG switch resonant wavelength of corrosion layer and the effective refractive index of neff. The simulation experiment use the optical fiber type of SMF-28TM which is used more commonly in communication, some specific parameters: $\mathrm{n}_{1}=1.46810, \mathrm{n}_{2}=1.46281$, $a=4.15 \mu \mathrm{m}$. Set the main structure parameters of corrosion FBG: the grating period: $\Lambda=528.93 \mathrm{~nm}$; corrosion zone diameters are respectively: $\mathrm{D}_{\mathrm{TH}}=9.3 \mu \mathrm{m}, 10.3$ $\mu \mathrm{m}, 12.3 \mu \mathrm{m}, 14.3 \mu \mathrm{m}, 16.3 \mu \mathrm{m}$; the range of SRI: 1.32 1.46 , then assuming that temperature is a constant. For a given optical parameters, solve the characteristic equation (2) when the wavelength $\lambda=1550 \mathrm{~nm}$, and then get the effective refractive index of neff. As shown in Figure 2 uniform corrosion of FBG in different cladding radius, there is a nonlinear relationship between the Fiber Bragg grating effective refractive index and changes of the external solution refractive index SRI.

The refractive index spectral shows that the sensitivity of corrosion area grating is associated with SRI directly. There exists a red-shift compared to the Bragg wavelength, when the external solution refractive index increases. Due to its fundamental guide model has a better restriction in this fiber core range, and resulting in a slight evanescent wave, when SRI is about 1.34 , the external refractive index has little effect on wavelength offset. When the external refractive index is close to the

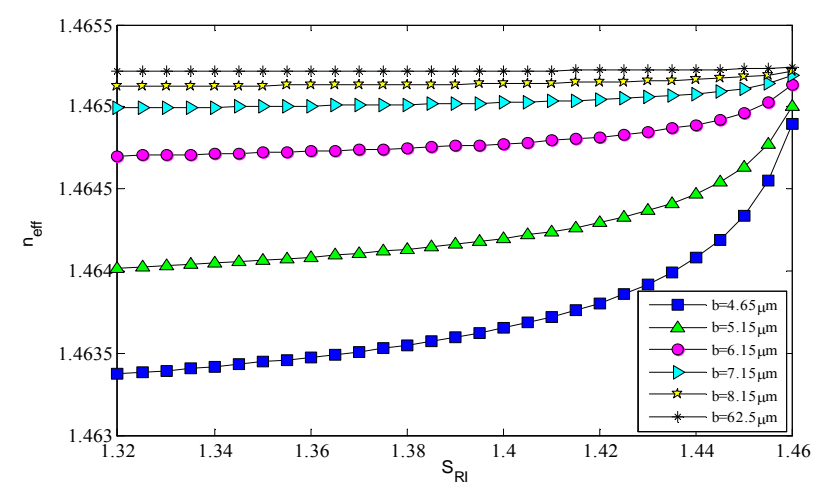

Figure 2. Different cladding radius effective index of fundamental mode with cladding refractive index changing. 
cladding refractive index $(\mathrm{SRI}=1.46)$, the offset reaches a maximum, at the same time the sensor reaches the maximum sensitivity; secondly, from (a) - (d) we can know that the smaller the thickness of the cladding layer, the higher the sensitivity of the sensor. So the relationship between the FBG wavelength and the change of the solution refractive index is obtained.(Figure 3)

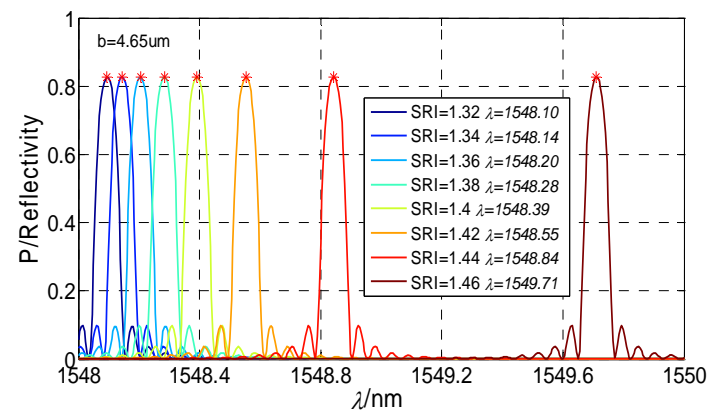

(a) $\mathrm{b}=4.65 \mu \mathrm{m}, \Delta \lambda=1.587 \mathrm{~nm}$

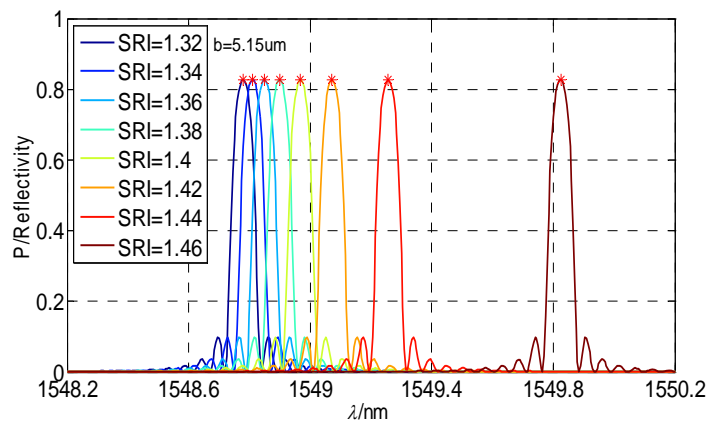

(b) $\mathrm{b}=5.15 \mu \mathrm{m}, \Delta \lambda=1.05786 \mathrm{~nm}$

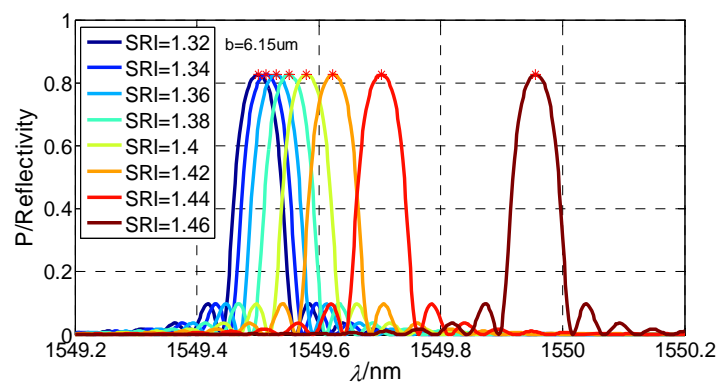

(c) $\mathrm{b}=6.15 \mu \mathrm{m}, \Delta \lambda=0.4231 \mathrm{~nm}$

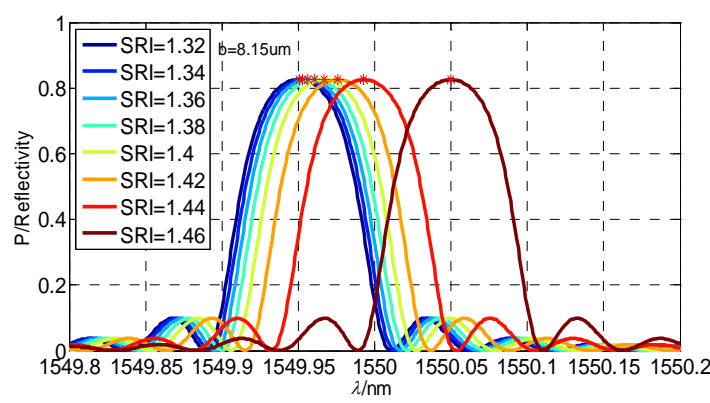

(d) $\mathrm{b}=8.15 \mu \mathrm{m}, \Delta \lambda=0.10579 \mathrm{~nm}$

Figure 3. The refractive index spectrum of FBG in different cladding radius.

\section{Experimental Analysis}

The measurement experiment structure diagram of the concentration sensor based on Fiber Bragg grating as follows: (Figure 4)

In order to study the sensitivity of the sensor, we select sucrose, Nacl, ethanol three solution for experiment, analysis the effects of different solutions at different concentrations (C less than $80 \%$ ) to the fiber grating wavelength, eventually complete the high precision measurement of the chemical concentration sensor based on Fiber Bragg grating.

The experimental data of three kinds of solution concentration $\mathrm{C}$ and refractive index can be measured by WAY-type Abbe refract meter, and combine the partial least squares, the linear expressions of the three kinds of solution are established. The expressions are as follows:

$\begin{array}{ll}\text { Sucrose } & \mathrm{n}=1.3285+0.00184 \mathrm{c} \\ \text { Nacl } & \mathrm{n}=1.3331+0.00185 \mathrm{c} \\ \text { Ethanol } & \mathrm{n}=1.3404+0.00033 \mathrm{c}\end{array}$

where, $\mathrm{C}$ is the solution quality percentage concentration, $\mathrm{n}$ is the refractive index. So the diagrams of the three kinds of solution concentration and refractive index are obtained. And it is shown in Figure 5. It can be seen from the figure, there are no significant differences between the values calculated and the true value of the refractive index of the three kinds of solution.

So when we take a fixed cladding radius, the diagram of the wavelength of FBG with the change of concentration of external solution obtained, through the relationship between external solution refractive index SRI and the effective refractive index of Fiber Bragg grating neff,

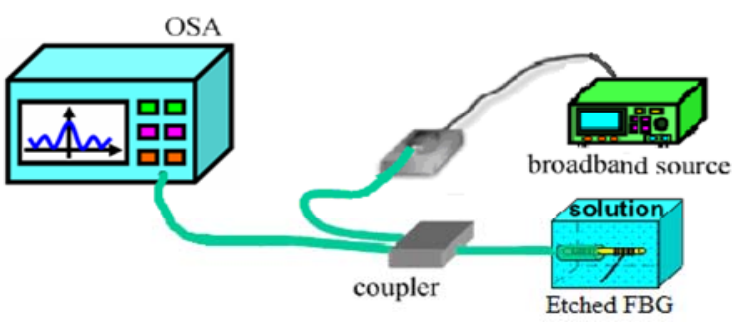

Figure 4. The experimental structure diagram.

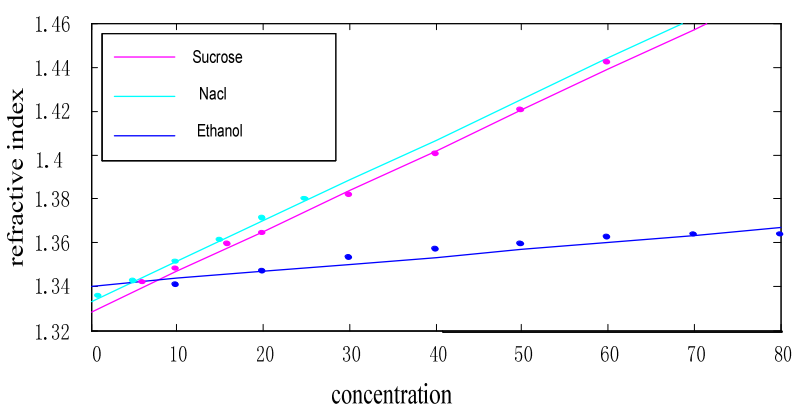

Figure 5. The correlation between concentration and refractive index for different solutions. 


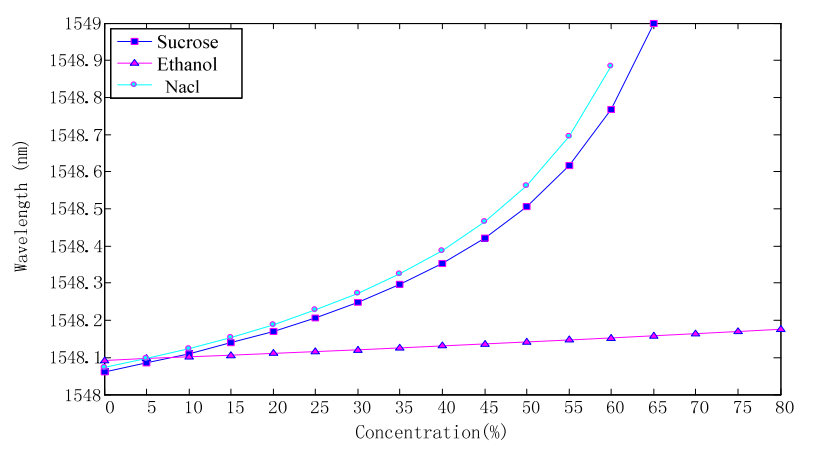

Figure 6. Relationshio between wavelength and sucrose solution concentration.

the relationship between the effective refractive index neff and resonant wavelength $\lambda_{B}$, and the relationship between concentration of solution $\mathrm{c}$ and its refractive index SRI.

Figure 6 shows that, when measuring the $0-80 \%$ concentration alcohol solution, the center wavelength of fiber grating changes a little with the change of the concentration of solution, and there has an approximate linear relation; when measuring Nacl and sucrose solution, the change of wavelength with solution concentration changing is obviously and show monotonically increasing nonlinear relation. The wavelength of the fiber grating sensor changes obviously when the concentration of Nacl changes. Therefore through the simulation we can know that the sensitivity of the sensor is the largest and the maximum sensitivity of the sensor is up to 185.59 $\mathrm{pm} / \mathrm{c} \%$.

Therefore the sensitivity of the sensor is the largest, from simulation we know that the maximum sensitivity of the sensor is up to $185.59 \mathrm{pm} / \mathrm{c} \%$.

\section{Conclusions}

The paper combines the optical waveguide theory, fiber grating coupling theory and evanescent field and partial least squares method theory. Though characteristics analysis and experimental of three-layer structure of the Fiber Bragg grating sensor accomplished. An experiment on the external solution concentration ( $\mathrm{c}=80 \%)$ is completed with the chemical sensors based on Fiber Bragg grating, the spectrum and sensitivity of different concentration of solution obtained. The sensor can be used to measure the liquid environment chemical or biological compositional changes, and can realize multi-point detection at the same time, thus possessing good application prospect in the filed of medical, pharmacy, petro- chemical industry, catering trade, etc.

\section{Acknowledgements}

This work is supported by the Fundamental Research Funds for The Northeastern University at Qinhuangdao. (XNK201308).

\section{REFERENCES}

[1] U. S. Raikar, A. S. Lalasangi, V. K. Kulkarni and J. F. Akki, "Concentration and Refractive Index Sensor for Methanol Using Short Period Grating Fiber," International Journal for Light and Electron Optics, Vol. 122, No. 1, 2011, pp. 89-91.

doi:10.1016/j.ijleo.2009.11.012

[2] Y. Zhan, J. Luo, H. Wu and M.H. Yu, "An All-Fiber High Resolution Fiber Grating Concentration Sensor," International Journal for Light and Electron Optics, Vol. 123, No.7, pp. 637-640

[3] Dionisio A. Pereira Orlando Fraza o. "Fiber Bragg Grating Sensing System for Simultaneous Measurement of Salinity and Temperature," Society of Photo-Optical Instrumentation Engineers, Vol. 43, No.2, 2004, pp. 299-304.

[4] M. F. Zhao, D.-Y. Huang, B. B. Luo, et al., "Evanescent Wave Sensors Based on Fiber Bragg Grating," Journal of Chongqing University of Technology, Natural Science, 2010, Vol. 24, No.10, pp. 46-50.

[5] T. M. Libish, J. Linesh, P. Biswas, S. Bandopadhyay, K. Dasgupta and P. Radhakrishnan. "Fiber Optic Long Period Grating Based Sensor for Coconut Oil Adulteration Detection," Sensors \& Transducers, 2010, Vol. 114, pp.102-111.

[6] U. S. Raikar, A. S. Lalasangi, Jyoti F. Akki, P. Raikar, et al. "Concentration Sensor Based on Fiber Grating Technology," Sensors and Actuators B: Chemical, Vol. 161, No.1,2012,pp. 818-823.doi:10.1016/j.snb.2011.11.039

[7] Q. Jiang, D. B. Hu and M. Yang, "Simultaneous Measurement of Liquid Level and Surrounding Refractive Index Using Tilted Fiber Bragg Grating," Sensors and Actuators A: Physical, 2011, pp. 1-4.

[8] W. S. Liu and Z. G. Guan, "Multiplexing of Fiber Bragg Grating Pairs for Sensing Based on Optical Low Coherence Technology," Microwave and Optical Technology letter, Vol. 50, 2008, pp. 2437-2439.

[9] M. F. Zhao, D. Y. Huang, B. Zhou and L. Z. Jiao, "Chemical Sensors Based on Fiber Bragg Grating," Mechanics and Materials, Vol. 84-85, 2011, pp. 582-585. doi:10.4028/www.scientific.net/AMM.84-85.582 